-Case Reports-

\title{
Anterior Cerebral Artery Dissection Presenting Subarachnoid Hemorrhage and Cerebral Infarction
}

\author{
Kentaro Suzuki, Masahiro Mishina, Seiji Okubo, Arata Abe, \\ Satoshi Suda, Masayuki Ueda and Yasuo Katayama \\ Department of Neurological, Nephrological and Rheumatological Science, Graduate School of Medicine, Nippon Medical School \\ Divisions of Neurology, Nephrology and Rheumatology, Department of Internal Medicine, Nippon Medical School Hospital
}

\begin{abstract}
A 35-year-old man presented with simultaneous occurrence of subarachnoid hemorrhage (SAH) and cerebral infarction (CI) caused by anterior cerebral artery (ACA) dissection. He complained of sudden onset of left frontal headache and his symptoms progressed to consciousness disturbance and right hemiparesis. Computed tomography and magnetic resonance imaging demonstrated SAH localized in the left interhemispheric fissure and $\mathrm{CI}$ in the territory of the left ACA. Right carotid angiography demonstrated a long double lumen sign at the left A2 to A4 segment of the left ACA, leading to a diagnosis of the combined type of $\mathrm{CI}$ and SAH caused by ACA dissection. Although many surgeons have previously tried to perform endovascular treatment, we selected only medication in this case, and his neurological findings gradually improved. Only 9 cases including the present case presented with simultaneous occurrence of SAH and CI caused by ACA dissection. Many of these patients showed stenosis with dilatation of ACA on carotid angiography. The prognoses of these patients were good. However, many SAH patients with dissecting aneurysm had poor prognoses. To improve the strategy for managing ACA dissection, we need to accumulate a greater number of such cases in the future. We also recommend that angiography should be performed in the patients with ACA dissection.
\end{abstract}

(J Nippon Med Sch 2012; 79: 153-158)

Key words: anterior cerebral artery, dissection, simultaneous, cerebral infarction, subarachnoid hemorrhage

\section{Introduction}

Infarction in the anterior cerebral artery (ACA) territory was first described in the early $20^{\text {th }}$ century based on autopsy results'. Recent studies using computed tomography (CT) and magnetic resonance imaging (MRI) have investigated the etiologies and clinical features. Cerebral infarcts localized in the ACA territory are reported to comprise $0.5-3 \%$ of all ischemic strokes $^{23}$. Spontaneous dissecting aneurysms of the internal carotid circulation occur frequently less than those of the vertebrobasilar circulation, with more than $90 \%$ of dissecting

Correspondence to Kentaro Suzuki, MD, Divisions of Neurology, Nephrology and Rheumatology, Department of Internal Medicine, Nippon Medical School, 1-1-5 Sendagi, Bunkyo-ku, Tokyo 113-8603, Japan

E-mail: kentarow@nms.ac.jp

Journal Website (http://www.nms.ac.jp/jnms/) 


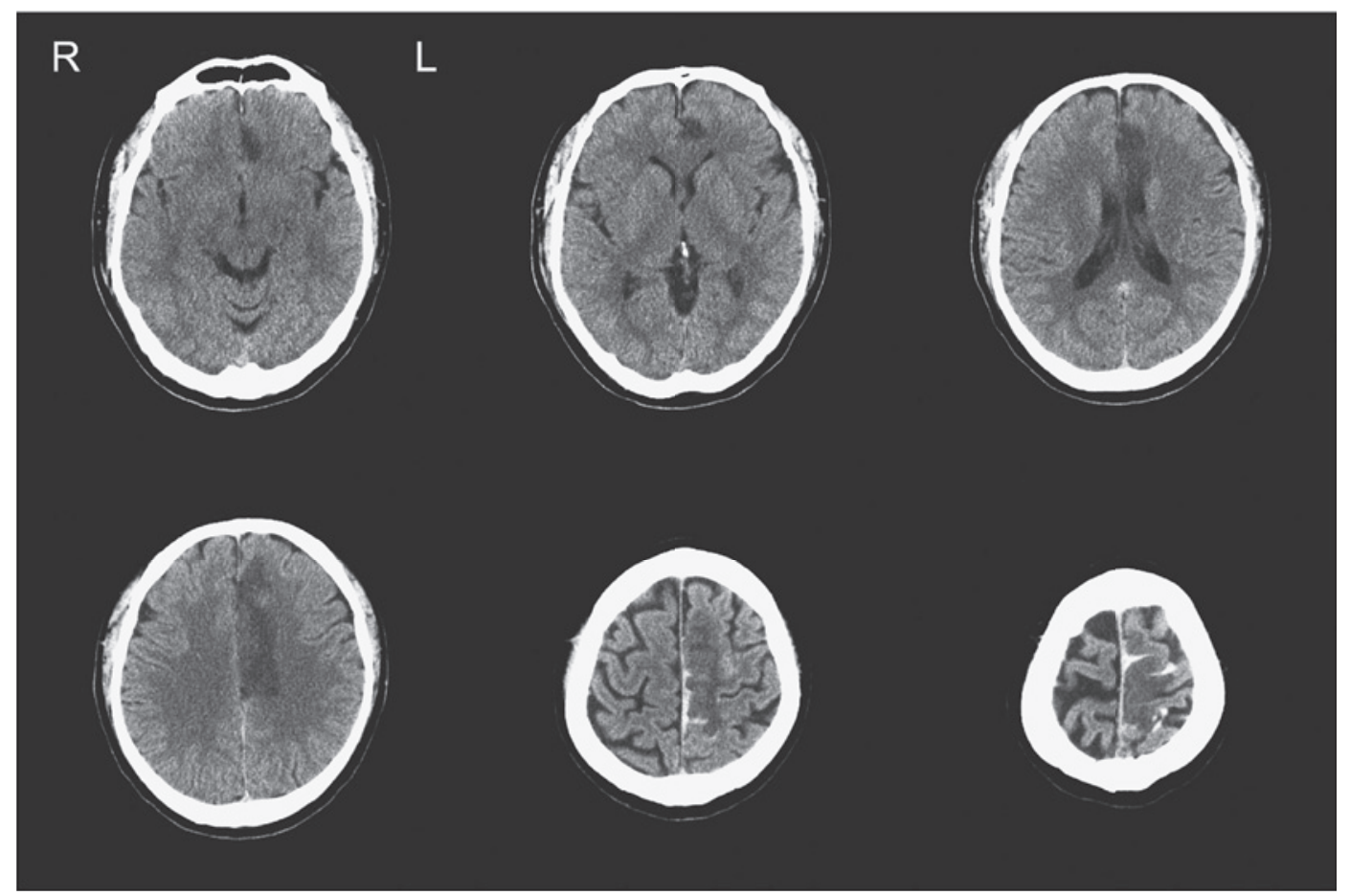

Fig. 1 Computed tomography on admission.

It is a computed tomography on admission at 9 hours after onset. High density areas were observed in the left interhemispheric fissure, and a low density area is observed in the territory of the left anterior cerebral artery.

aneurysms occurring in the vertebrobasilar circulation $^{4}$. ACA dissection is reported to comprise $36.7 \%$ of intracranial dissections ${ }^{5}$. Spontaneous intracranial dissection often develops in young healthy people and is associated with severe symptom. We report a case of simultaneous cerebral infarction and subarachnoid hemorrhage (SAH) caused by dissection of the ACA. It was a rare case, but was an important case considering the management of younger stroke.

\section{Case Report}

A 35-year-old male patient complained of sudden onset of left frontal headache during work. The headache worsened gradually, and he was admitted to a hospital. After seven hours, however, his symptoms progressed to consciousness disturbance and right hemiparesis. Then he was referred to our hospital. He had a habit of smoking 30 cigarettes a day, and a history of hypertension without medication. He did not have a history of head injury.

On admission, he demonstrated aphasia, right facial palsy, hemiparesis and sensory disturbance. The National Institute of Health Stroke Scale (NIHSS) was 20. Systolic blood pressure was 148 $\mathrm{mmHg}$ and diastolic blood pressure was $100 \mathrm{mmHg}$. General laboratory data were normal. Initial computed tomography at 9 hours after onset demonstrated SAH localized in the left interhemispheric fissure, and cerebral infarction in the territory of the left ACA (Fig. 1). In MRI fluid attenuation inversion recovery (FLAIR) image also showed high intensity signals, and $\mathrm{T} 2{ }^{*}$-weighted image showed low intensity signals in the left interhemispheric fissure (Fig. 2). FLAIR, T2weighted and diffusion-weighted image (DWI) MRI showed cerebral infarct in the territory of the left ACA (Fig. 2). Left carotid angiography (CAG) demonstrated a long double lumen sign at the left A2 to A4 segment one day after onset (Fig. 3). We diagnosed the combined type of cerebral infarction and SAH caused by ACA dissection.

We administered nicardipine, glycerol and edaravone for the treatment of cerebral infarction ${ }^{6-8}$. The patient's neurological findings gradually 


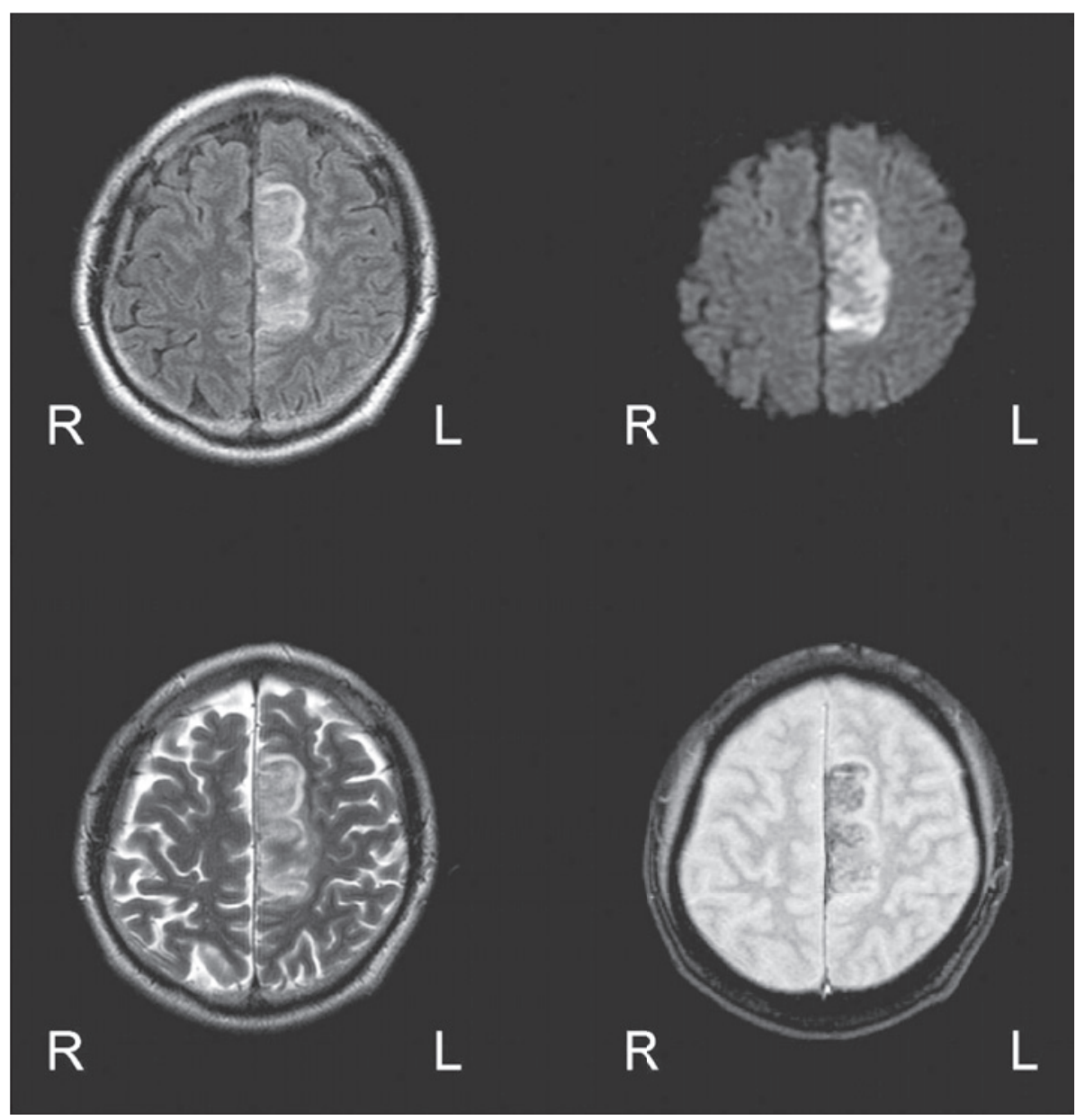

Fig. 2 Magnetic resonance imaging.

In the left interhemispheric fissure, we detected high intensity signals on fluid attenuation inversion recovery (FLAIR) image (upper left) and low intensity signals on T2*-weighted image (lower right). FLAIR, T2-weighted (lower left) and diffusionweighted images (upper right) showed a high intensity area in the territory of left anterior cerebral artery.

improved on day 5. We could not find any new lesion on MRI, and right CAG did not show enlargement of ACA dissection on day 10. Then the patient started walk rehabilitation. NIHSS had improved to 10 on day 14 and 2 at 3 months after the onset. Modified Rankin Scale was 1 at 3 months.

\section{Discussion}

Shimoyama et al. indicated that spontaneous intracranial arterial dissection (SICAD) was found in $64.3 \%$ of patients with ACA infarction. SICAD is relatively infrequent among patients with middle cerebral artery (MCA) or posterior cerebral artery infarction ${ }^{9}$. There are two reasons to explain this difference. First, embolism in the ACA may be unlikely because the diameter of the A1 segment is approximately half that of the M1 segment and cerebral blood flow is lower in the ACA than in the $\mathrm{MCA}^{3}$. Second, atherosclerotic changes are frequently found in the $\mathrm{MCA}^{10}$. According to the Spontaneous Cervicocephalic Arterial Dissections Study (SCADS), 'Double lumen' demonstrated on DSA is defined as the major criterion of cervicocephalic arterial dissection ${ }^{11}$. Our patient demonstrated a long 'double lumen' in the CAG, and we could diagnose the patient as having arterial dissection. Regarding pathological features, a subintimal dissection tends to result in stenosis of the arterial lumen, leading to cerebral infarctions, whereas a subadventitial dissection may cause aneurysmal dilatation of the artery, resulting in 


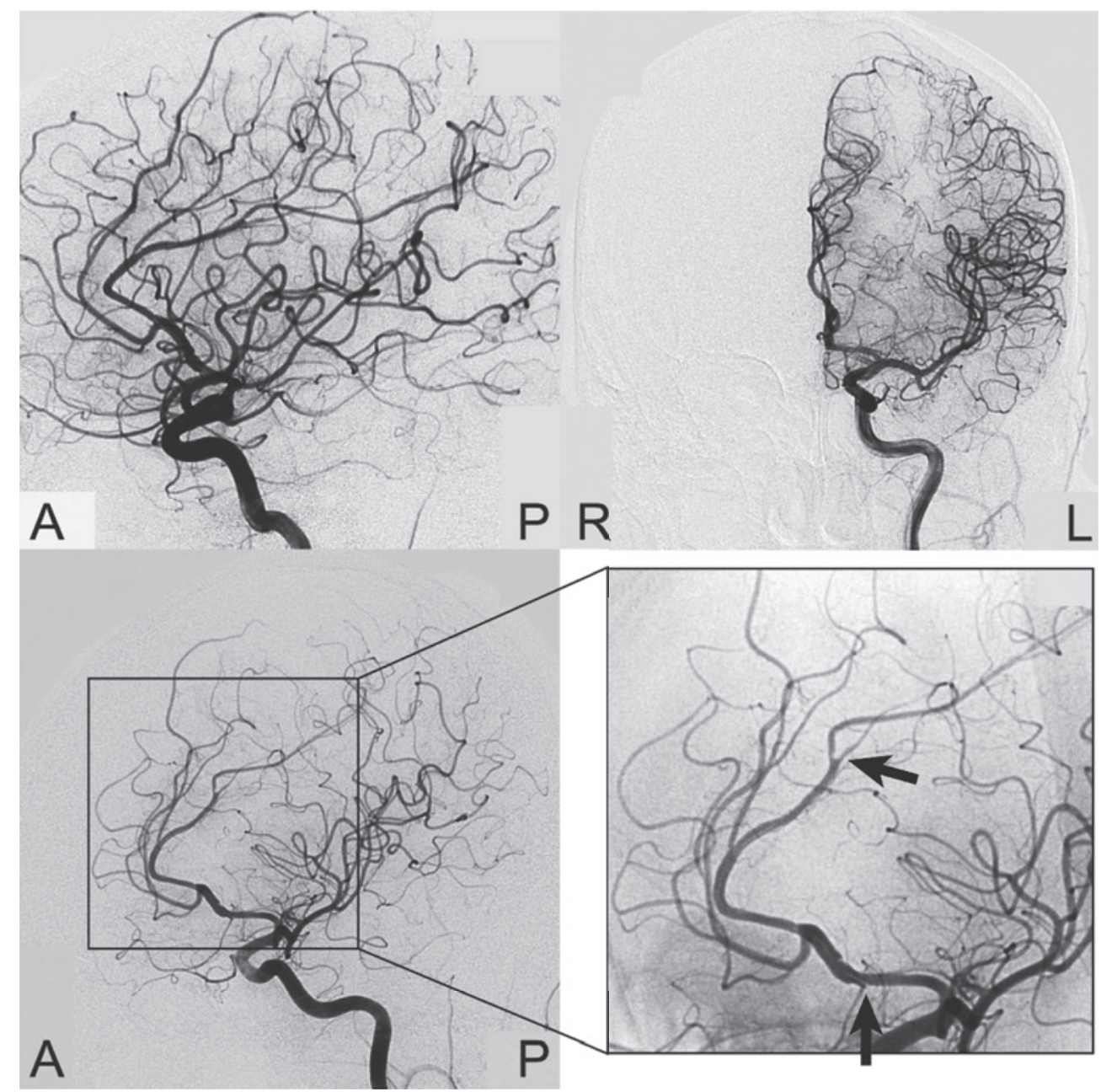

Fig. 3 Right carotid angiography showing lateral view (upper left), anteroposterior view (upper right), left anterior oblique (LAO) $30^{\circ}$ (lower left) and magnified image of LAO (lower right).

Long double lumen sign was observed in the A2 to A4 segment of the left anterior cerebral artery (from the lower arrow to the upper arrow). In the A2 segment, dilatation of the artery was also found.

hemorrhagic events. Seven of 9 cases of ACA dissection with simultaneous subarachnoid hemorrhage and infarction showed stenosis with dilatation (Table 1). In these cases, we estimated to occur the both mechanisms. Many of the patients with ACA dissection accompanied by simultaneous $\mathrm{SAH}$ and infarction showed nonperimesencephalic SAH. Generally, a good outcome of nonaneurysmal nonperimesencephalic SAH has been reported $^{12}$. Therefore, we consider that all cases of ACA dissection with $\mathrm{SAH}$ and infarction showed a relative good outcome comparison with dissection with SAH, because it is not easy to demonstrated perimesencephalic lesion by ACA dissection.

Sato et al. indicated that patients with stroke in the ACA territory caused by dissection were younger and less commonly had a history of heart disease or previous stroke than patients without dissection $^{13}$. Anterior cerebral artery dissection in 9 patients who simultaneously developed $\mathrm{SAH}$ and cerebral infarction including the present case showed similar characteristics, such as good outcome and middle age (Table 1$)^{14-18}$. In the case of Inoue et al., a 48-year-old man was complained of sudden onset of headache and left hemiparesis. MRI and $\mathrm{CT}$ detected dissection from $\mathrm{A} 1$ to $\mathrm{A} 2$, and demonstrated SAH and $\mathrm{CI}$ in the ACA territory. The patient initially treated by edaravone, but digital subtraction angiography (DSA) confirmed the enlarged fusiform dilatation of the right A2 with 
Table 1 Cases of simultaneous subarachnoid hemorrhage and infarction caused by dissection of the anterior cerebral artery

\begin{tabular}{lcccc}
\hline \multicolumn{1}{c}{ Authors } & Age/Sex & Site of Lesion & Angiographic findings & outcome \\
\hline Yasukawa et al. (1993) & 62/M & Lt. A2 & stenosis with dilatation & good recovery \\
Kato et al. (2000) & $40 / \mathrm{F}$ & Lt. A2 & stenosis with dilatation & good recovery \\
Miyahara et al. $(2001)^{16}$ & $50 / \mathrm{M}$ & Rt. A2-A3 & stenosis with dilatation & good recovery \\
Ohkuma et al. $(2003)^{17}$ & $40 / \mathrm{M}$ & A2 & stenosis without dilatation & good recovery \\
Ohkuma et al. $(2003)^{17}$ & $64 / \mathrm{F}$ & A2 & stenosis with dilatation & good recovery \\
Ohkuma et al. $(2003)^{17}$ & $49 / \mathrm{F}$ & A2 & stenosis with dilatation & good recovery \\
Ohkuma et al. $(2003)^{17}$ & $49 / \mathrm{M}$ & A3 & stenosis without dilatation & good recovery \\
Inoue et al. (2010) & $49 / \mathrm{M}$ & ACoA-rt. A2 & stenosis with dilatation & good recovery \\
Present case & 35/M & Lt. A2-A3 & stenosis with dilatation & good recovery \\
\hline
\end{tabular}

$\mathrm{ACo} \mathrm{A}=$ anterior communicating artery.

distal narrowing on day 14 . So endvascular trapping was performed. The patient remained neurologically stable after operation and got an outpatient with mild monoparesis of the left lower extremity eight month after the onset.

Because the prognoses are different, it is very important to distinguish ischemic or hemorrhagic disease early. The optimal treatment for ACA dissection remains controversial. The onset of ischemia is generally indicated for conservative therapy, whereas the onset of bleeding almost always requires surgical treatment. In this case, because vasodilatation was limited, we selected conservative treatment by administration of edaravone without surgery and achieved a good outcome. A Japanese multi-center study showed that a novel free radical scavenger, edaravone, was efficacious in patients with acute ischemic stroke ${ }^{1920}$. In vitro and in vivo studies showed that edaravone inhibited brain edema after ischemia ${ }^{21.22}$, delayed neuronal death ${ }^{23}$, and vascular endothelial cell injur $^{24}$. Specifically, because edaravone in the membrane can promote the delivery of fat soluble vitamin $\mathrm{E}$ inside the membrane and water soluble vitamin $\mathrm{C}$ outside the membrane and effectively prevent damage to membrane lipids, which are susceptible to radical injury, we suggest that edaravone is not complicating factor for arterial dissection.

Recently, we could easily diagnose such cases as ACA dissection with advanced technology such as DSA and MRI. Because treatment differs based on pathophysiology of the stroke type and many SAH patients with dissecting aneurysm had poor prognoses, prompt and accurate diagnosis is importan $\mathrm{t}^{25}$. To improve the strategy for managing ACA dissection, we need to accumulate a greater number of ACA dissection cases in the future. We also recommend that angiography be performed in patients with ACA dissection.

\section{References}

1. Critchley M: The anterior cerebral artery and its syndromes. Brain 1930; 53: 120-165.

2. Kang SY, Kim JS: Anterior cerebral artery infarction: stroke mechanism and clinical-imaging study in 100 patients. Neurology 2008; 70: 2386-2393.

3. Kazui S, Sawada T, Naritomi H, Kuriyama Y, Yamaguchi T: Angiographic evaluation of brain infarction limited to the anterior cerebral artery territory. Stroke 1993; 24: 549-553.

4. Hosoya T, Adachi M, Yamaguchi K, Haku T, Kayama T, Kato T: Clinical and neuroradiological features of intracranial vertebrobasilar artery dissection. Stroke 1999; 30: 1083-1090.

5. Ohkuma H, Suzuki S, Ogane K: Dissecting aneurysms of intracranial carotid circulation. Stroke 2002; 33: 941-947.

6. Mishina M, Komaba Y, Kobayashi S, et al.: Efficacy of edaravone, a free radical scavenger, for the treatment of acute lacunar infarction. Neurologia medico-chirurgica 2005; 45: 344-348.

7. Mishina M, Komaba Y, Kobayashi S, et al.: Administration of free radical scavenger edaravone associated with higher frequency of hemorrhagic transformation in patients with cardiogenic embolism. Neurologia medico-chirurgica 2008; 48: 292-297.

8. Shinohara Y, Saito I, Kobayashi S, Uchiyama S: Edaravone (radical scavenger) versus sodium ozagrel (antiplatelet agent) in acute noncardioembolic ischemic stroke (EDO trial). Cerebrovasc Dis 2009; 27: 485-492.

9. Shimoyama T, Kimura K, Iguchi $\mathrm{Y}$, et al.: 
Spontaneous intra-cranial arterial dissection frequently causes anterior cerebral artery infarction. Journal of the neurological sciences 2011; 304: 40-43.

10. Caplan LR, Gorelick PB, Hier DB: Race, sex and occlusive cerebrovascular disease: a review. Stroke; a journal of cerebral circulation 1986; 17: 648-655.

11. Minematsu K, Matsuoka H, Kasuya J, SCADS-I collaborators: Cervicocepharic Arterial Dissections in Japan: Analysis of 454 patients in the Spontaneous Cervicocephalic Arterial Dissections Study I (SCADS-I). Stroke 2008; 39: 566.

12. Gupta SK, Gupta R, Khosla VK, et al:: Nonaneurysmal nonperimesencephalic subarachnoid hemorrhage: is it a benign entity? Surgical neurology 2009; 71: 566-571.

13. Sato S, Toyoda K, Matsuoka $\mathrm{H}$, et al.: Isolated anterior cerebral artery territory infarction: dissection as an etiological mechanism. Cerebrovascular diseases 2010; 29: 170-177.

14. Yasukawa K, Kamijo Y, Ikeda A: A case of anterior cerebral artery dissecting aneurysm presenting subarachnoid hemorrhage and cerebral infarction at the same time. Surgery for Cerebral Stroke 1993; 21: 461-466.

15. Kato N, Yamada Y, Hyodo A, Nose T: A case of anterior cerebral artery dissecting presenting subarachnoid hemorrhage and cerebral infarction. Jpn J Neurosurg 2000; 9: 157-161.

16. Miyahara K, Sakata K, Gondo G, Kanno H, Yamamoto I: Spontaneous dissection of the anterior cerebral artery presenting subarachnoid hemorrhage and cerebral infarction: a case report. Neurological surgery 2001; 29: 335-339.

17. Ohkuma H, Suzuki S, Kikkawa T, Shimamura N: Neuroradiologic and clinical features of arterial dissection of the anterior cerebral artery. AJNR. American journal of neuroradiology 2003; 24: 691699.

18. Inoue $\mathrm{T}$, Fujimura $\mathrm{M}$, Matsumoto $\mathrm{Y}$, Kondo $\mathrm{R}$, Shimizu H, Tominaga T: Simultaneous occurrence of subarachnoid hemorrhage and cerebral infarction caused by anterior cerebral artery dissection treated by endovascular trapping. Neurologia medicochirurgica 2010; 50: 574-577.

19. Abe K, Yuki S, Kogure K: Strong attenuation of ischemic and postischemic brain edema in rats by a novel free radical scavenger. Stroke; a journal of cerebral circulation 1988; 19: 480-485.

20. Edaravone Acute Infarction Study Group: Effect of a novel free radical scavenger, edaravone (MCI-186), on acute brain infarction. Randomized, placebocontrolled, double-blind study at multicenters. Cerebrovascular diseases 2003; 15: 222-229.

21. Watanabe T, Yuki S, Egawa M, Nishi H: Protective effects of MCI-186 on cerebral ischemia: possible involvement of free radical scavenging and antioxidant actions. The Journal of pharmacology and experimental therapeutics 1994; 268: 1597-1604.

22. Nishi H, Watanabe T, Sakurai H, Yuki S, Ishibashi A: Effect of MCI-186 on brain edema in rats. Stroke; a journal of cerebral circulation 1989; 20: 1236-1240.

23. Yamamoto T, Yuki S, Watanabe T, Mitsuka M, Saito KI, Kogure K: Delayed neuronal death prevented by inhibition of increased hydroxyl radical formation in a transient cerebral ischemia. Brain research 1997; 762: 240-242.

24. Watanabe T, Morita I, Nishi H, Murota S: Preventive effect of MCI-186 on 15-HPETE induced vascular endothelial cell injury in vitro. Prostaglandins, leukotrienes, and essential fatty acids 1988; 33: 81-87.

25. Takumi I, Mizunari T, Mishina M, et al.: Dissecting posterior inferior cerebellar artery aneurysm presenting with subarachnoid hemorrhage right after anticoagulant and antiplatelet therapy against ischemic event. Surgical Neurology 2007; 68: 103-107.

(Received, August 11, 2011)

(Accepted, October 11, 2011) 